Article

\title{
An Improved, Negatively Correlated Search for Solving the Unit Commitment Problem's Integration with Electric Vehicles
}

\author{
Qun Niu ${ }^{1, *}$, Kecheng Jiang ${ }^{1}$ and Zhile Yang ${ }^{2}$ (I) \\ 1 Shanghai Key Laboratory of Power Station Automation Technology, School of Mechanical Engineering and \\ Automation, Shanghai University, Shanghai 200444, China; 18817639961@163.com \\ 2 Shenzhen Institute of Advanced Technology, Chinese Academy of Sciences, Shenzhen 518055, China; \\ zyang07@qub.ac.uk \\ * Correspondence: nq@shu.edu.cn
}

Received: 29 September 2019; Accepted: 26 November 2019; Published: 5 December 2019

check for updates

\begin{abstract}
With the rapid development of plug-in electric vehicles (PEVs), the charging of a number of PEVs has already brought huge impact and burden to the power grid, particularly at the medium and low voltage distribution networks. This presents a big challenge for further mass roll-out of electric vehicles. To assess the impact of charging of substantial number of electric vehicles on the grid, a model of 30000 PEVs integrated with unit commitment (UCEV) was investigated in this study. The unit commitment was a large-scale, mixed-integer, nonlinear, NP-Hard (non-deterministic polynomial) optimization problem, while the integration of PEVs further increased the complexity of the model. In this paper, a global best inspired negatively correlated search (GBNCS) method which extends the evolutionary logic of negatively correlated search is proposed to tackle the UCEV problem. In the proposed algorithm, a rounding transfer function in GBNCS, is deployed to convert real-valued variables into binary ones; further, the global best information is combined in the population to improve the efficiency of the algorithm. Numerical results confirmed that the proposed GBNCS can achieve good performance in both a basic IEEE 10 unit commitment problem and the UCEV problem. It was also shown that, among four charging modes, the off-peak charging mode and EPRI (Electric Power Research Institute) charging mode are more economical in PEV charging.
\end{abstract}

Keywords: unit commitment; electric vehicle; negatively correlated search

\section{Introduction}

Industry revolutions and urbanization have led to a number of intractable environmental and resource problems, such as global warming, due to extensive consumption of fossil fuels [1]. According to a research by the U.S. Energy Information Administration (EIA) in 2014, carbon emission is the single most important factor for the global warming and the transportation sector accounts for more than $1 / 3$ of the total greenhouse gas emissions [2]. Therefore, to reduce the carbon emissions from the transportation sector is an important part of the global effort to curb global warming. To achieve this goal, many governments have introduced a wide range of policies to promote the development of electric vehicles.

Generally speaking, electric vehicles can be grouped into three categories: battery electric vehicles (BEVs), hybrid electric vehicles (HEVs), and plug-in electric vehicles (PEVs). Due to the technical limitations of fuel cells and the huge costs in the production and storage of hydrogen, the fuel cell electric vehicles are still not suitable for large-scale applications, while HEVs have already been widely used. In recent years, the advances in the battery technology and power control [3] have further improved 
the efficiency of HEVs. But they still use fossil fuels. With the maturity of battery technology, lithium batteries, nickel metal hydride batteries, and lead-acid batteries have been widely used in electric vehicles [4-6], and the PEV has become the most popular EV type. Both the research and practice have confirmed the great potentials of PEVs in energy conservation and emission reduction $[7,8]$.

However, the rapid growth of the electric vehicle market has introduced new problems and challenges. The charging of a massive number of electric vehicles is likely to have a huge impact and burden to the power grid, which is another problem that needs to be addressed $[9,10]$. Further, questions such as how to save the operational costs while integrating the charging of EVs and how to cope with stochastic charging behavior of different EV owners should be answered. In [11], Zhou et al. presented a probability model of EV charging load in the distribution network. In [12], the EV charging model was studied from the demand side, and the charging demand data was collected from a real application in Korea and the load control strategy was employed to develop a smart charging technique suitable for practical applications. In this paper, in order to investigate the effect of EV charging, the charging of EVs is combined with the unit commitment problem. The charging demand is formulated as an unpredictable load to the power system which needs to be addressed for power secure operation at the lowest cost.

The unit commitment problem (UC) is a large-scale, mixed-integer, nonlinear, NP-Hard (non-deterministic polynomial) optimization problem. The aim of unit commitment is to minimize the generation costs by reasonably scheduling the on/off status and output power of generations under various system constraints. Conventional methods such as dynamic programming [13] and lagrangian relaxation [14] are able to quickly solve low dimension UC problems. But these two methods both have poor performance and may not guarantee to find the optimum solutions in large-scale UC problems [15]. In the last few decades, various methods, including artificial neural networks [16], Tabu search [17], simulated annealing [18], branch and cut (BC) [19], Benders decomposition (BD) [20], and intelligent meta-heuristic algorithms, such as particle swarm optimization [21] and a genetic algorithm [15], have been applied to solve the UC problem. However, the complexity of the UC problem is significantly increased due to the introduction of new participants such as electrical vehicles.

To handle the increased complexity, Saber proposed an intelligent unit commitment model with vehicle-to-grid (V2G) and applied binary particle swarm optimization to find a balance in reducing both fuel costs and emissions [22]. But the paper does not consider the stochastic charging scenario and uncertainties associated with charging and discharging. To investigate the impact of EV charging in different situations, Foley proposed two charging modes; namely, peak charging mode and off-peak charging mode [23]. Ma et al. further combined Foley's EV charging mode witha multi-objective, dynamic, economic, emission load dispatch problem [24]. In Liu's research, a stochastic unit commitment model with plug-in electric was combined with wind power [25]. Mohammadi designed a solar-powered EV which reduces the use of fossil cells and can be combined into the research of optimization problems in power systems such as UC [26]. Yang et al. further proposed a framework to comprehensive study the unit commitment problem integrating various renewable generations and PEVs [27]. Further, bidirectional power charging control strategy is also an important research direction which can help reduce the burden to the power grid [28,29]. In this paper, four charging modes, including the peak charging mode, the off-peak charging mode, the Electric Power Research Institute (EPRI) charging mode, and a stochastic charging mode are combined with the UC problem to thoroughly investigate the effect of EV charging on the power system.

The negatively correlated search (NCS) was first proposed by Tang et al. [30] and has shown to deliver excellent performance for continuous optimization problems. The core logic of NCS is divide-and-conquer; the search domain is divided into several parts and each part is a random local search (RLS) which helps to avoid trapping into local optimum. However, NCS is rarely applied to binary or mixed-integer optimization problems such as UC. Furthermore, the computation time of the search process is too long, due to the calculation of correlations between individuals. Therefore, a binary, negatively correlated search (BNCS) is proposed in this paper which uses a rounding 
transfer function to convert real-valued variables into binary ones. To improve the efficiency of BNCS, the global best solution is introduced to BNCS, leading to the proposal of the global best inspired binary negatively correlated search (GBNCS) algorithm. These two algorithms were first tested on eight benchmark instances of the knapsack problem and then applied to UC problems.

The remainder of the paper is organized as follows: Section 2 presents the formulation of the unit commitment problem, followed by the proposed global-best inspired negative correlated search (GBNCS) and its implementation for a UC problem in Section 3, and the numerical results and analysis are given in Section 4. Finally, Section 5 concludes the paper.

\section{Problem Formulation}

The unit commitment problem, including the basic unit commitment and the unit commitment with electrical vehicles can be formulated as follows.

\subsection{Basic Unit Commitment}

The unit commitment problem can be modeled as a kind of optimization problem which aims at minimizing the objective function while equality and inequality constraints at the system level such as power balance limit and minimum up/down time limit have to be met.

\subsubsection{Objective Function}

The objective function of basic unit commitment problem consists of two parts including the fuel cost and the start-up cost.

\section{Fuel cost}

The committed units in power system usually have different power and efficiency. To dispatch these units economically, the fuel cost becomes a significant factor which can be expressed by a quadratic equation.

$$
F_{f c, i}\left(P_{u, i}^{t}\right)=a_{i}+b_{i} P_{u, i}^{t}+c_{i}\left(P_{u, i}^{t}\right)^{2}
$$

where $F_{f c, i}$ and $P_{u, i}^{t}$ present the fuel cost and the power of $i$ th unit at time $t$ respectively, and $a_{i}, b_{i}$, and $c_{i}$ are the fuel cost coefficients associated to the $i$ th unit.

\section{Start-up cost}

The start-up cost is also an important part of the objective function which can be divided into cold start-up cost and hot start-up cost. If the unit has not been off for too long time, it may take less time and cost to start up, whereas if the unit has been off for enough time, it may take much longer to start up the unit. The start-up cost related to unit is given by:

$$
f_{s c, n}=\left\{\begin{array}{l}
C_{n}^{\text {hot }} \quad \text { if } \quad M_{n}^{\text {down }} \leq T_{n}^{\text {off }} \leq M_{n}^{\text {down }}+C H_{n} \\
C_{n}^{\text {cold }} \quad \text { if } T_{n}^{\text {off }}>M_{n}^{\text {down }}+C H_{n},
\end{array}\right.
$$

where $C_{n}^{\text {hot }}$ is the hot start-up cost and $C_{n}^{\text {cold }}$ is the cold start-up cost. $M_{n}^{\text {down }}$ is the minimum down time, $T_{n}^{o f f}$ is the off-line duration time, and $\mathrm{CH}_{n}$ is the cold-start hour of $n$th unit.

Therefore, the objective function of the unit commitment problem combining both the fuel cost and start-up cost can be expressed as follows, where $U_{i}^{t}$ is a binary variable representing the on/off state of $i$ th unit at time $t$, and $U_{i}^{t-1}$ represents the on/off state of the $i$ th generating unit at time $t-1$.

$$
\min F=\sum_{t=1}^{T} \sum_{i=1}^{N}\left[\left(F_{f c, i}\left(P_{u, i}^{t}\right)\right)+F_{s c, i}\left(1-U_{i}^{t-1}\right)\right] \cdot U_{i}^{t}
$$




\subsubsection{Constraints}

In the following, various constraints that need to be considered at the unit commitment stage are presented to ensure the adequacy of the system after implementation, while other constraints which are often considered at lower system operation and control levels relating to power system adequacy, contingency, vulnerability, and stability, are not included.

\section{Power balance constraint}

The power generated by all the units at time $t$ needs to meet the power demand, which is given in Equation (4), where $P d(t)$ is the demand power, and $P_{i}^{t}$ is the generation power of unit $i$ at time $t$.

$$
\sum_{i=1}^{N} P_{i}^{t} U_{i}^{t}=P d(t)
$$

\section{Generation limit}

The power of each unit should be within its rated generation range, as defined in Equation (5), where $P_{i}^{\min }$ and $P_{i}^{\max }$ respectively represent the minimum and maximum power outputs of the $i$ th unit.

$$
P_{i}^{\min } \leq P_{i}^{t} \leq P_{i}^{\max }
$$

\section{Minimum up and minimum down time constraint}

To maintain the unit in good condition, the generation unit should not be turned on or off too frequently and has to meet the following constraints.

$$
\left\{\begin{array}{l}
T_{n}^{o f f}>M_{n}^{\text {down }} \\
T_{n}^{o n}>M_{n}^{u p},
\end{array}\right.
$$

where $T_{n}^{o f f}$ and $T_{n}^{o n}$ represent the off-line time and on-line time of unit $n ; M_{n}^{u p}$ and $M_{n}^{\text {down }}$ represent the minimum up/down time of unit $n$.

\section{Ramp rates constraint}

The power output of each unit cannot be changed violently, and it has to meet the ramp rate constraint given below,

$$
P_{i}^{\min }(t) \leq P_{i}^{t} \leq P_{i}^{\max }(t),
$$

where $P_{i}^{\min }(t)$ and $P_{i}^{\max }(t)$ in Equation (7) are the minimum and maximum outputs of unit $i$ at time $t$, which are defined as follows, where $R U_{i}$ and $R D_{i}$ are the ramp-up/down rates of the $i$ th unit and $P_{i}^{t-1}$ representing the output of unit $i$ at time $t-1$.

$$
\begin{aligned}
& P_{i}^{\min }(t)=\max \left(P_{i}^{t-1}-R D_{i}, P_{i}^{\min }\right) \\
& P_{i}^{\max }(t)=\min \left(P_{i}^{t-1}+R U_{i}, P_{i}^{\max }\right)
\end{aligned}
$$

\section{Spinning reserve constraint}

To meet with the uncertainty of power demand forecast and unexpected failure of generating units, the spinning reserve constraint is considered, which is given by,

$$
\sum_{i=1}^{N} P_{i}^{\max } U_{i}^{t}-P d(t) \cdot(1+S R) \geq 0
$$


where $P_{i}^{\max }$ is the maximum output of $i$ th unit, $P d(t)$ is the power demand at $t$ time, and $S R$ is the spinning reserve factor for controlling the reserve capacity of the system.

\subsection{Unit Commitment Integrated with EV}

The unit commitment integrated with electric vehicles model is based on the basic unit commitment problem and the objective function associated with the fuel cost and start-up cost remains the same as before, but the integration of electric vehicles introduces new and more complex constraints.

\subsubsection{Constraints of UC Combined with EVs}

\section{Power Balance Constraint}

The charging load is added to the demand load due to additional load of EVs charging. Therefore, the power balance constraint in the unit commitment with EVs is given by:

$$
\sum_{i=1}^{N} P_{i}^{t} U_{i}^{t}-P_{c a r}(t)=P d(t)
$$

where $P_{c a r}(t)$ is the additional load for charging electric vehicles at time $t$.

\section{Spinning reserve constraint}

In order to solve the instantaneous demand changes brought by electric vehicle charging, the reserved capacity needs to increase.

$$
\sum_{i=1}^{N} P_{i}^{\max } U_{i}^{t}+P_{c a r}^{\max }(t) \geq P d(t) \cdot(1+S R)
$$

where $P_{c a r}^{\max }(t)$ is the maximum power required for electric vehicle charging.

\subsubsection{Different Charging Models}

Since different EV users often have different charging habits which increase the complexity and stochasticity of the problem, four charging modes were introduced to reflect different charging portfolios; those included the off-peak charging mode (OPCM), peak charging mode (PCM), Electric Power Research Institute Charging Mode (EPRICM) and the stochastic charging mode (SCM).

\section{Off-peak charging mode (OPCM)}

In off-peak charging mode users usually charge EVs between 23:00 to 6:00; in this period of time the demand power is usually not high and the impact on the grid caused by the charging of EV is not significant. Foley et al. assumed a plain load curve to simulate the $10 \%$ EV charging load curve in Ireland in 2013 [23]. According to [23], research on the probability distribution of $24 \mathrm{~h}$ off-peak charging mode given in Table 1 and Figure 1 represents the probability distribution more intuitively.

Table 1. The probability of charging electric vehicles (EVs) in off-peak mode.

\begin{tabular}{ccccccc}
\hline Time Period & \multicolumn{6}{c}{ Charging Probability } \\
\hline $01: 00-06: 00$ & $18.5 \%$ & $18.5 \%$ & $9 \%$ & $9 \%$ & $4 \%$ & $4 \%$ \\
$07: 00-12: 00$ & $0 \%$ & $0 \%$ & $0 \%$ & $0 \%$ & $0 \%$ & $0 \%$ \\
$13: 00-18: 00$ & $0 \%$ & $0 \%$ & $0 \%$ & $0 \%$ & $0 \%$ & $0 \%$ \\
$19: 00-24: 00$ & $0 \%$ & $0 \%$ & $0 \%$ & $0 \%$ & $18.5 \%$ & $18.5 \%$ \\
\hline
\end{tabular}




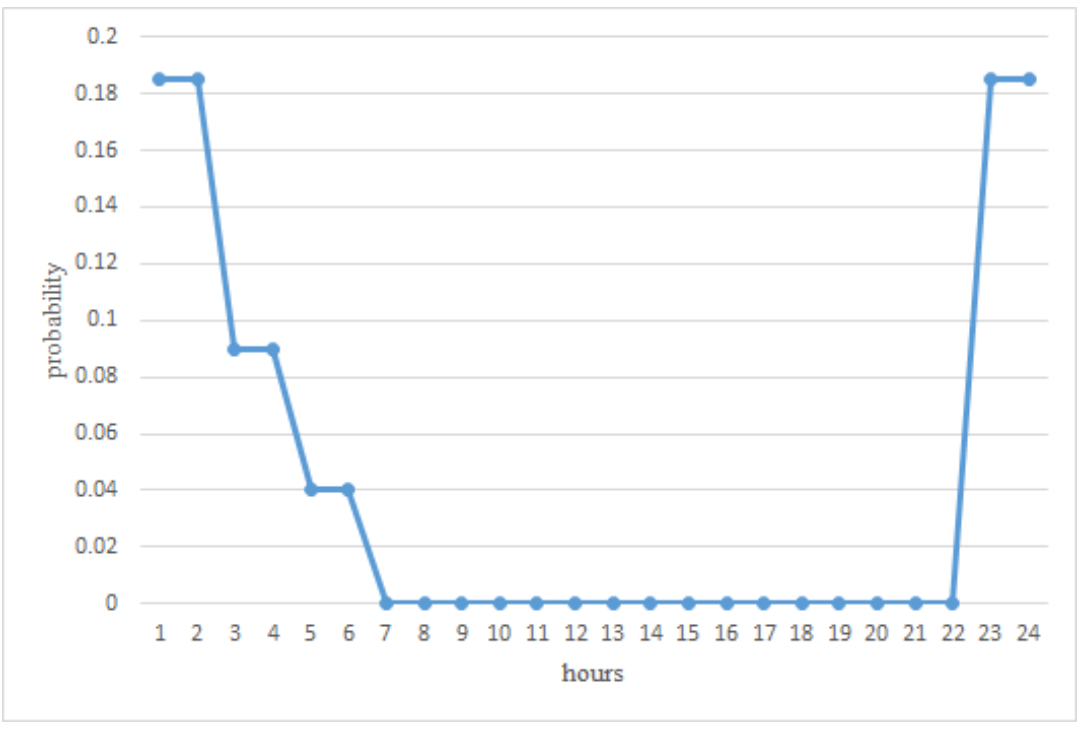

Figure 1. The probability of charging EVs in off-peak mode.

\section{Peak charging mode (PCM)}

Different from the off-peak charging mode, the peak charging mode considers the most extreme situations. In the peak charging Mode, it is assumed that almost all EVs are charged during peak hours, which will bring a huge impact and burden to the power system. Table 2 and Figure 2 [23] give the probability distribution of $24 \mathrm{~h}$ peak charging Mode; the charging peak is mainly concentrated during the period from 13:00 to 20:00.

Table 2. The probability of charging EVs in peak mode.

\begin{tabular}{ccccccc}
\hline Time Period & \multicolumn{7}{c}{ Charging Probability } \\
\hline $01: 00-06: 00$ & $0 \%$ & $0 \%$ & $0 \%$ & $0 \%$ & $0 \%$ & $0 \%$ \\
$07: 00-12: 00$ & $0 \%$ & $0 \%$ & $0 \%$ & $0 \%$ & $9 \%$ & $9 \%$ \\
$13: 00-18: 00$ & $18.5 \%$ & $18.5 \%$ & $18.5 \%$ & $18.5 \%$ & $0 \%$ & $0 \%$ \\
$19: 00-24: 00$ & $4 \%$ & $4 \%$ & $0 \%$ & $0 \%$ & $0 \%$ & $0 \%$ \\
\hline
\end{tabular}

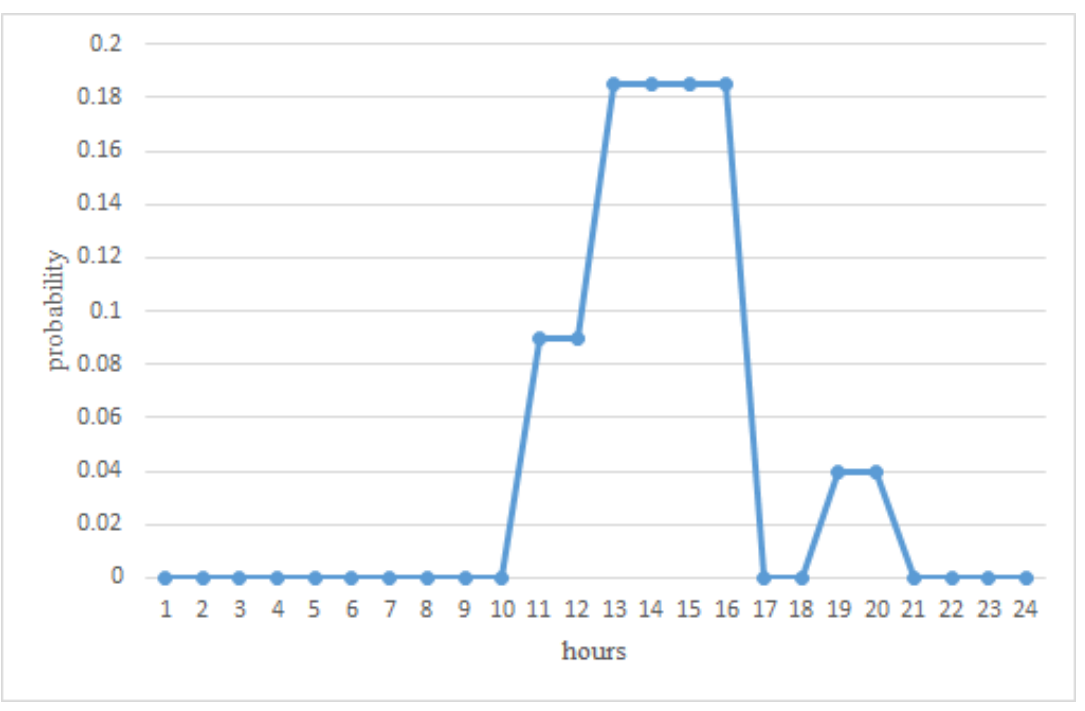

Figure 2. The probability of charging EVs in PEAK mode. 


\section{Electric Power Research Institute Charging Mode (EPRICM)}

The Electric Power Research Institute Charging Mode (EPRICM) is an electric vehicle charging mode proposed by the Electric Power Research Institute (EPRI). The research work of EPRI in 2007 [31] integrated the $24 \mathrm{~h}$ daily charging probability distribution of electric vehicles in the United States, which is shown in Table 3, and the fitting curve of this model is illustrated in Figure 3. It can be concluded from the curve that three-quarters of the EVs are charged between 23:00 and 6:00, and the remaining EVs are charged at other periods.

Table 3. The probability of charging EVs in Electric Power Research Institute Charging Mode (EPRI) mode.

\begin{tabular}{ccccccc}
\hline Time Period & \multicolumn{6}{c}{ Charging Probability } \\
\hline $01: 00-06: 00$ & $10 \%$ & $10 \%$ & $9.5 \%$ & $7 \%$ & $5 \%$ & $3 \%$ \\
$07: 00-12: 00$ & $1 \%$ & $0.3 \%$ & $0.3 \%$ & $1.3 \%$ & $2 \%$ & $2 \%$ \\
$13: 00-18: 00$ & $2 \%$ & $2 \%$ & $2 \%$ & $1 \%$ & $0.3 \%$ & $0.3 \%$ \\
$19: 00-24: 00$ & $1.5 \%$ & $3 \%$ & $5 \%$ & $9.5 \%$ & $10 \%$ & $10 \%$ \\
\hline
\end{tabular}

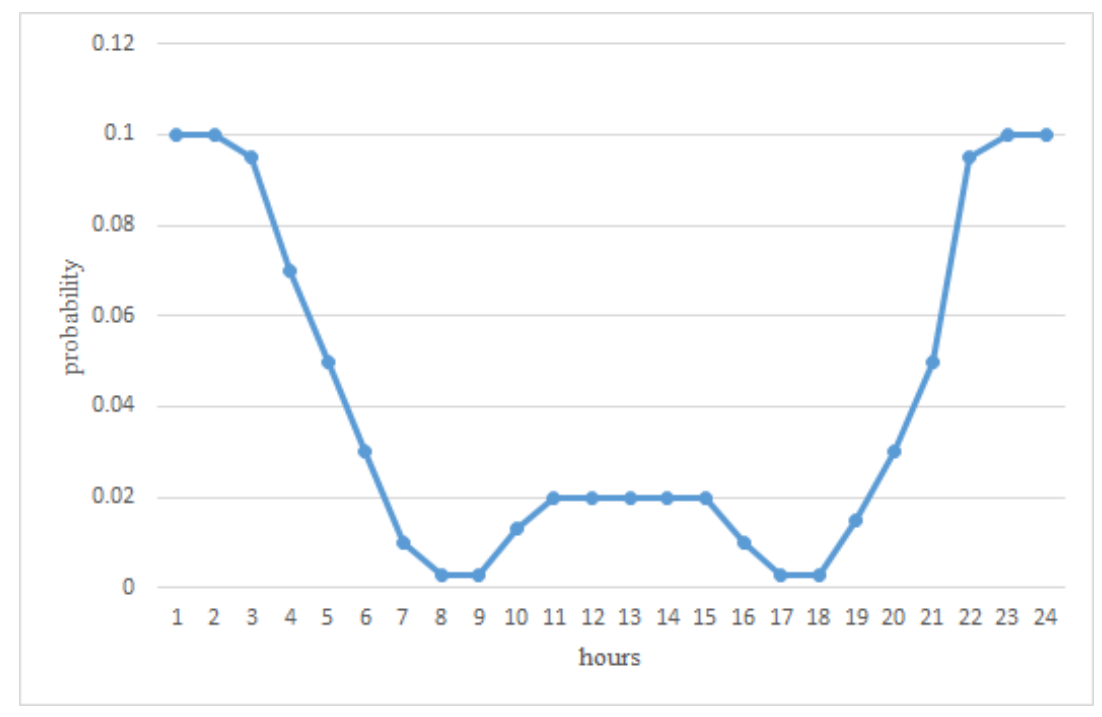

Figure 3. The probability of charging EVs in EPRI mode.

\section{Stochastic charging mode (SCM)}

The off-peak charging mode and the peak charging Mode are usually assumed with ideal conditions. However, due to different charging habits, users of electric vehicles often change with daily activities, resulting in stochastic charging periods and charging duration. Therefore, the stochastic charging mode may be closer to the actual charging situation. In stochastic charging mode, five scenarios are randomly generated by a Gaussian operator $N(\lambda, \sigma)$ where the mean value $\lambda$ and standard deviation $\sigma$ are respectively set as $4.16 \%(1 / 24)$ and 0.01 . The probability distribution of five cases is given in Table 4 and Figure 4. 
Table 4. The probability of charging EVs in stochastic mode.

\begin{tabular}{|c|c|c|c|c|c|c|}
\hline \multirow{2}{*}{$\begin{array}{c}\text { Time Period } \\
\text { 01:00-06:00 }\end{array}$} & \multicolumn{6}{|c|}{ Charging Probability (Scenario1) } \\
\hline & $5.70 \%$ & $4.90 \%$ & $4.80 \%$ & $2.40 \%$ & $2.60 \%$ & $9.70 \%$ \\
\hline 07:00-12:00 & $8.70 \%$ & $4.80 \%$ & $1.10 \%$ & $3.20 \%$ & $2.10 \%$ & $5.70 \%$ \\
\hline 13:00-18:00 & $3.80 \%$ & $2.20 \%$ & $2.10 \%$ & $6.10 \%$ & $3.20 \%$ & $2.20 \%$ \\
\hline 19:00-24:00 & $2.80 \%$ & $2.20 \%$ & $5.50 \%$ & $2.50 \%$ & $3.50 \%$ & $8.20 \%$ \\
\hline Time period & \multicolumn{6}{|c|}{ Charging probability (scenario2) } \\
\hline 01:00-06:00 & $9.98 \%$ & $5.81 \%$ & $6.92 \%$ & $2.04 \%$ & $3.22 \%$ & $3.62 \%$ \\
\hline $07: 00-12: 00$ & $6.36 \%$ & $3.60 \%$ & $5.56 \%$ & $0.06 \%$ & $3.45 \%$ & $2.51 \%$ \\
\hline 13:00-18:00 & $1.01 \%$ & $5.18 \%$ & $4.72 \%$ & $4.23 \%$ & $1.49 \%$ & $6.41 \%$ \\
\hline 19:00-24:00 & $4.86 \%$ & $3.56 \%$ & $4.21 \%$ & $3.64 \%$ & $3.97 \%$ & $3.59 \%$ \\
\hline Time period & \multicolumn{6}{|c|}{ Charging probability (scenario3) } \\
\hline 01:00-06:00 & $3.40 \%$ & $3.96 \%$ & $6.53 \%$ & $2.78 \%$ & $5.42 \%$ & $4.65 \%$ \\
\hline $07: 00-12: 00$ & $3.79 \%$ & $2.47 \%$ & $3.71 \%$ & $4.02 \%$ & $2.84 \%$ & $4.47 \%$ \\
\hline 13:00-18:00 & $2.84 \%$ & $4.01 \%$ & $4.70 \%$ & $2.71 \%$ & $3.70 \%$ & $4.72 \%$ \\
\hline 19:00-24:00 & $1.22 \%$ & $5.82 \%$ & $8.04 \%$ & $5.07 \%$ & $3.65 \%$ & $4.85 \%$ \\
\hline Time period & \multicolumn{6}{|c|}{ Charging probability (scenario4) } \\
\hline 01:00-06:00 & $3.10 \%$ & $4.58 \%$ & $2.47 \%$ & $5.82 \%$ & $3.07 \%$ & $8.16 \%$ \\
\hline $07: 00-12: 00$ & $2.32 \%$ & $4.51 \%$ & $1.57 \%$ & $2.91 \%$ & $3.15 \%$ & $4.84 \%$ \\
\hline 13:00-18:00 & $5.76 \%$ & $4.67 \%$ & $3.53 \%$ & $5.55 \%$ & $5.52 \%$ & $4.36 \%$ \\
\hline 19:00-24:00 & $5.13 \%$ & $4.86 \%$ & $2.48 \%$ & $5.45 \%$ & $3.04 \%$ & $3.13 \%$ \\
\hline Time period & \multicolumn{6}{|c|}{ Charging probability (scenario5) } \\
\hline 01:00-06:00 & $4.35 \%$ & $4.91 \%$ & $4.33 \%$ & $8.90 \%$ & $2.18 \%$ & $1.01 \%$ \\
\hline 07:00-12:00 & $2.22 \%$ & $2.30 \%$ & $3.42 \%$ & $3.87 \%$ & $3.79 \%$ & $5.08 \%$ \\
\hline 13:00-18:00 & $4.82 \%$ & $5.44 \%$ & $7.18 \%$ & $6.24 \%$ & $1.98 \%$ & $0.77 \%$ \\
\hline 19:00-24:00 & $5.69 \%$ & $1.04 \%$ & $4.27 \%$ & $4.22 \%$ & $7.95 \%$ & $4.04 \%$ \\
\hline
\end{tabular}

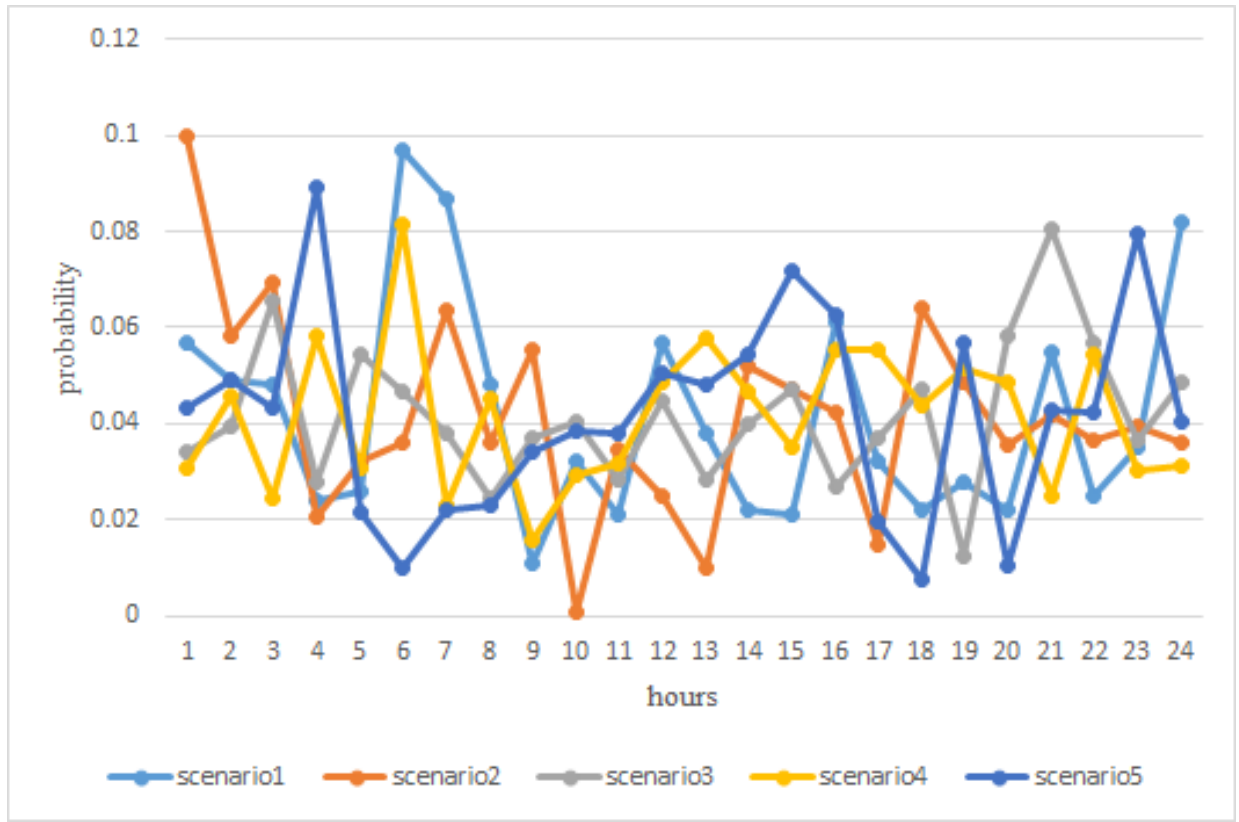

Figure 4. The probability of charging EVs in stochastic mode. 


\section{Negatively Correlated Search}

\subsection{Negatively Correlated Search}

Negatively correlated search (NCS) is a population based algorithm proposed by Tang [30]. The core logic of NCS is automatic divide-and-conquer, and it is different from conventional population based evolutionary algorithms, such as genetic algorithm (GA), which use stochastic recombination.

When solving a minimization optimization problem, GA concentrates more on stochastic recombination. During the evolution process, crossover and mutation operator are applied to the algorithm to obtain better individuals which have smaller objective value, and in the later selection phase, these individuals have a greater probability of being selected. Stochastic recombination shares the useful information between individuals and combines this information to help improve the efficiency of the algorithm. But there are also drawbacks for stochastic recombination. Since the generation of new individuals always combines the information from the existing individuals, the evolution of the population will easily converge prematurely and the solutions are trapped into local optimum during the evolution process. In order to overcome the premature convergence problem, NCS uses automatic divide-and-conquer which divides the population into several subpopulations; each subpopulation is ordered to search different parts among the feasible domain. Automatic divide and conquer improves the global search ability of the algorithm, helps NCS jump out of local optimum, and finds global optimum. To simplify the algorithm, it is assumed that each subpopulation only contains one individual and the search process of this individual is a randomized local search (RLS).

The Bhattacharyya distance [32] is defined to evaluate the divisibility of two discrete probability distributions in statistics. In NCS, the Bhattacharyya distance used to measure the difference between individuals is defined in the following equation, where $p_{i}$ and $p_{j}$ are the probability density functions of two distributions.

$$
D_{B}\left(p_{i}, p_{j}\right)=-\ln \left(\int \sqrt{p_{i}(x) p_{j}(x)} d x\right) .
$$

Hence, the correlation between RLSs is defined as follows:

$$
\operatorname{Corr}\left(p_{i}\right)=\min \left\{D_{B}\left(p_{i}, p_{j}\right) \mid j \neq i\right\} .
$$

Equation (14) formulates the search process of a RLS. A gausses mutation operator is employed to generate a new solution, where $x_{i d}$ is the $d t h$ variable of an existing solution; $N\left(0, \sigma_{i}\right)$ gives a random number with zero mean and sigma standard deviation.

$$
x_{i d}^{\prime}=x_{i d}+N\left(0, \sigma_{i}\right) .
$$

Only one solution will be selected from either the original one or the new one in a RLS; the selected solution should not only have smaller objective value $\mathrm{f}(\mathrm{x})$ but should also be distant from other RLSs which reflect in a larger $\operatorname{Corr}(p)$. The ratio of $f(x)$ and $\operatorname{Corr}(p)$ is defined to combine these two factors into the selection criterion as given below, where $\lambda$ is a parameter used for balancing the importance of $f(x)$ and $\operatorname{Corr}(p) . f\left(x^{\prime}\right)$ is the objective value of new solution and $\operatorname{Corr}\left(p^{\prime}\right)$ is the correlation between other RLSs.

$$
x_{n e w, i}=\left\{\begin{aligned}
x_{i} & \text { if } \frac{f\left(x_{i}^{\prime}\right)}{\operatorname{Corr}\left(p_{i}^{\prime}\right)}<\lambda \\
x_{i}^{\prime} & \text { otherwise. }
\end{aligned}\right.
$$




\subsection{Global Best Inspired Binary Negatively Correlated Search}

The NCS has been proven to have good performance in continuous optimization problem. However, the original NCS is not suitable for practical binary optimization problems. In [33], a binary negatively correlated search(BNCS) has been proposed to enable NCS to solve binary optimization problems [33]. A more efficient global best inspired binary negatively correlated search (GBNCS) is proposed in this paper. In BNCS, a rounding transfer function is used to convert the real valued variables into binary ones which are given in Equation (16).

$$
x_{\text {binary }}= \begin{cases}1 & \text { if } x<0.5 \\ 0 & \text { otherwise. }\end{cases}
$$

The global best inspired binary negatively correlated search (GBNCS) algorithm is improved based on the BNCS algorithm; the best solution in the population is employed in the selection phase of GBNCS; when the search process is trapped into local optimum, solutions distant from the best solution are preferred. The information shared by the best solution not only helps the search process jump out of the local optimum, but also improves the convergence. Here, this algorithm is named global best inspired binary negatively search (GBNCS). Different from BNCS, hamming distance [34] is used in GBNCS to measure the distance from the best solution which is widely used in evaluating the correlation between binary vectors. The definition of hamming distance is given by:

$$
H_{i j}=\sum_{d=1}^{n}\left|x_{i k}-x_{j k}\right|,
$$

where $x_{i d}$ and $x_{j d}$ are the $d t h$ variables of two different solutions and $H_{i j}$ is the hamming distance between these two solutions.

Hence, the selection strategy is changed as defined in Equation (18). Different from original NCS and BNCS, the correlation between individuals $\operatorname{Corr}\left(p_{i}^{\prime}\right)$ is replaced by the hamming distance between each individual and the best individual $H_{g i}$.

$$
x_{n e w, i}= \begin{cases}x_{i} & \text { if } \frac{f\left(x_{i}^{\prime}\right)}{H_{g i}}<\lambda \\ x_{i}^{\prime} & \text { otherwise. }\end{cases}
$$

To improve the efficiency of the algorithm, adaptive $\lambda$ and $\sigma$ was applied to the algorithm in Tang's work [30], which is given in Equation (19), where iter is the index of current iteration, $T_{\max }$ is the maximum iteration number, and $\lambda_{\text {iter }}$ stands for the value of $\lambda$ in iteration iter.

$$
\lambda_{\text {iter }}=N\left(1,0.1-0.1 * \frac{i t e r}{T_{\max }}\right) .
$$

A commonly used, self-adaptive step size, namely, the 1/5 successful rule proposed by Rechenberg [35], is applied-which is defined in Equation (20), where $\sigma_{i}$ is the step size, $r$ is a number less than 1 , and epoch is the frequency of the step size adjustment.

$$
\sigma_{i}= \begin{cases}\frac{\sigma_{i}}{r} & \text { if } \frac{c}{\text { epoch }}>0.2 \\ \sigma_{i} * r & \text { if } \frac{c}{\text { epoch }}<0.2 \\ \sigma_{i} & \text { if } \frac{c}{\text { epoch }}=0.2 .\end{cases}
$$

The flowchart of the GBNCS is given in Figure 5. 


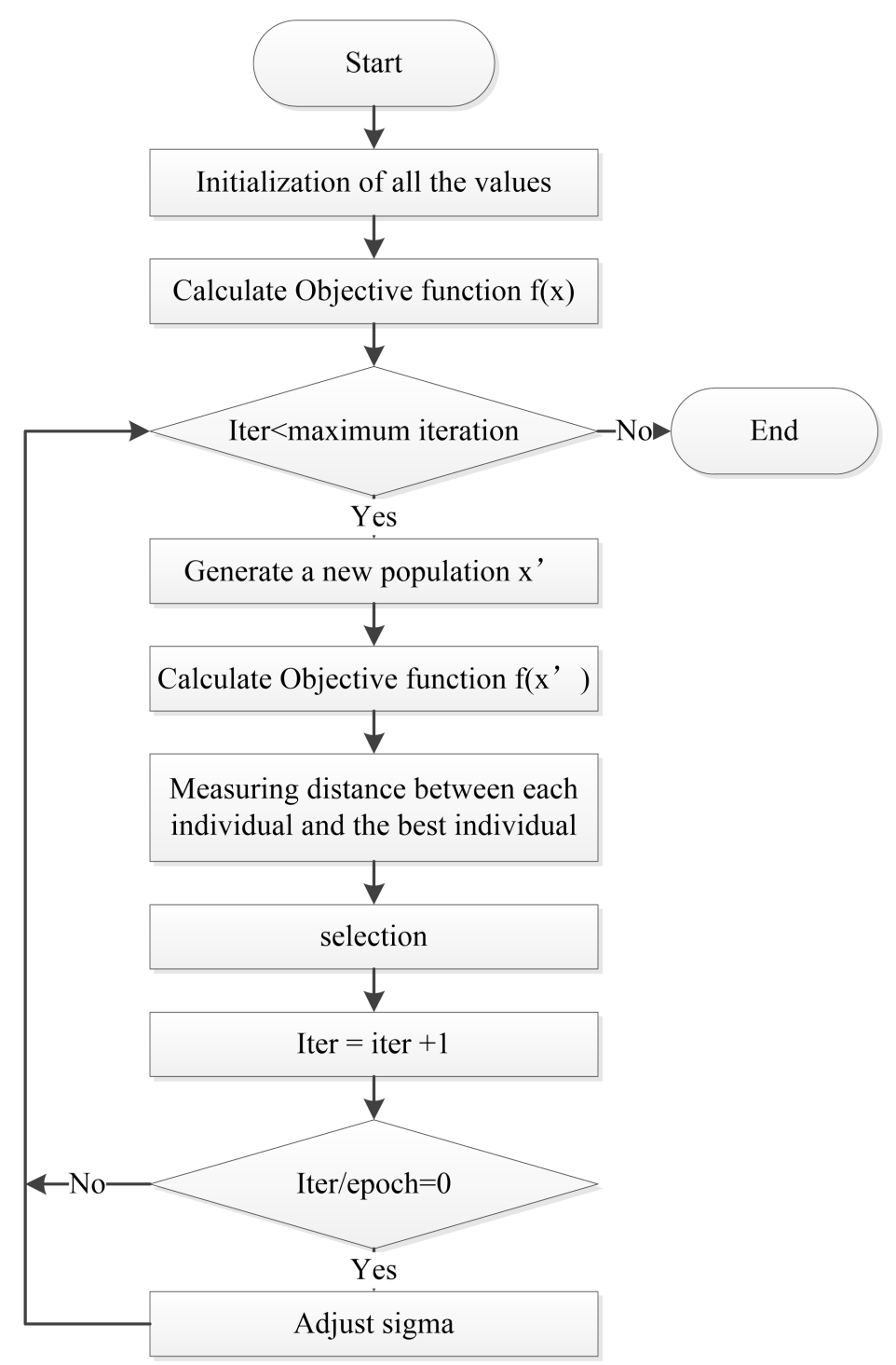

Figure 5. Optimization procedure of global best inspired binary negative correlate search.

3.3. The Proposed Algorithm for UC Problem

The main procedure based on the GBNCS to solve UC problem is presented as follows:

Step 1 Initialize the parameters including the step size $\sigma$, epoch, $\lambda, r$, Max_FES (total number of cost function evaluations), and population size.

Step 2 Randomly generate a population consisting binary variables $(0,1)$ representing the on/off status of units.

Step 3 Handle the minimum up/down time and spinning reserve constraints by adjusting the population.

Step 4 Solve the economic load dispatch (ELD) sup-problem, where a lambda iteration method is used to solve this problem [36].

Step 5 Calculate the objective function using Equation (3).

Step 6 While stopping criterion is not met,

Step 6.1 Generate a new population using Gaussian mutation operator according to Equation (14), and convert the variables to binary variables based on Equation (16). 
Step 6.2 Handle the minimum up/down time and spinning reserve constraints by adjusting the population.

Step 6.3 Solve the economic load dispatch (ELD) sub-problem, where a lambda iteration method is used to solve this problem.

Step 6.4 Calculate the objective function of the new population.

Step 6.5 Calculate the Hamming distance between each solution and the best solution.

Step 6.6 Select the solution with better quality using Equation (18).

Step 7 End while.

\section{Experimental Results}

In this section, the proposed BNCS and GBNCS is first tested on the knapsack problem [37], and then applied to the basic UC problem and the UC problem integrated with the EV problem. The experiments were conducted using MATLAB2016a on an Intel Core i7-8700k CPU with $3.7 \mathrm{GHz}$, a personal computer with 32 GB of RAM. The unit data and load demand, which were obtained from the IEEE-10-unit system, are given in Table 5 [15], and the spinning reserve was assumed to be $10 \%$ of the demand.

\subsection{Knapsack Problem}

The knapsack problem is a classical, binary, NP-hard optimization problem which has many practical applications in many fields, such as the finance, engineering, and computing. The Knapsack problem can be modeled as a maximum optimization problem given by Equation (21), where $w_{i}$ and $c_{i}$ represent the quality and value of ith item respectively, and $M$ is the maximum capacity of the backpack. The aim is to get the maximum total value of the items without exceeding the capacity of the backpack.

$$
\begin{aligned}
& \max f(X)=\sum_{i=1}^{n} c_{i} x_{i} \\
& \text { s.t. } \sum_{i=1}^{d} w_{i} x_{i} \leq M .
\end{aligned}
$$

Although the knapsack problem has already been thoroughly studied, the benchmark functions of the knapsack problem are still often used to test the performance of binary algorithms. In this paper, ten benchmark functions of the knapsack problem are used to test the performance of BNCS and GBNCS. The dimension and parameters of ten test problems are listed in Table 6 [37]. To test the convergence of these algorithms, the total number of cost function evaluations (FES) was set as 10,000.

Table 5. The characteristics of units in the 10-unit base system.

\begin{tabular}{ccccccccccc}
\hline Parameters & U1 & U2 & U3 & U4 & U5 & U6 & U7 & U8 & U9 & U10 \\
\hline$P_{\max }(M W)$ & 455 & 455 & 130 & 130 & 162 & 80 & 85 & 55 & 55 \\
$P_{\min }(M W)$ & 150 & 150 & 20 & 20 & 25 & 20 & 25 & 10 & 10 & 10 \\
$a(\$ / h)$ & 1000 & 970 & 700 & 680 & 450 & 370 & 480 & 660 & 665 & 670 \\
$b(\$ / M W h)$ & 16.19 & 17.26 & 16.6 & 16.5 & 19.7 & 22.26 & 27.74 & 25.92 & 27.27 & 27.79 \\
$c\left(\$ / M W h^{2}\right)$ & 0.00048 & 0.00031 & 0.002 & 0.00211 & 0.00398 & 0.00712 & 0.00079 & 0.00413 & 0.00222 & 0.00173 \\
$M U T(h)$ & 8 & 8 & 5 & 5 & 6 & 3 & 3 & 1 & 1 & 1 \\
$M D T(h)$ & 8 & 8 & 5 & 5 & 6 & 3 & 3 & 1 & 1 & 1 \\
$S U_{H}(\$$ & 4500 & 5000 & 550 & 560 & 900 & 260 & 260 & 30 & 30 & 30 \\
$S U_{C}(\$$ & 9000 & 10000 & 1100 & 1120 & 1800 & 520 & 520 & 60 & 60 & 60 \\
$T_{\text {cold }}(h)$ & 5 & 5 & 4 & 4 & 4 & 2 & 2 & 0 & 0 & 0 \\
Initial state $(\mathrm{h})$ & 8 & 8 & -5 & -5 & -6 & -3 & -3 & -1 & -1 & -1 \\
\hline
\end{tabular}


It is shown from the results in Table 6 that GBNCS produces the best performance of the two algorithms. The GBNCS is always able to find the best solution of almost all test cases except for F8 in $10,000 \mathrm{FES}$, and the BNCS's performance is not ideal due to the slow convergence. Figure 6 gives the converge curve in different dimensions of F1, F2, F5, and F8.
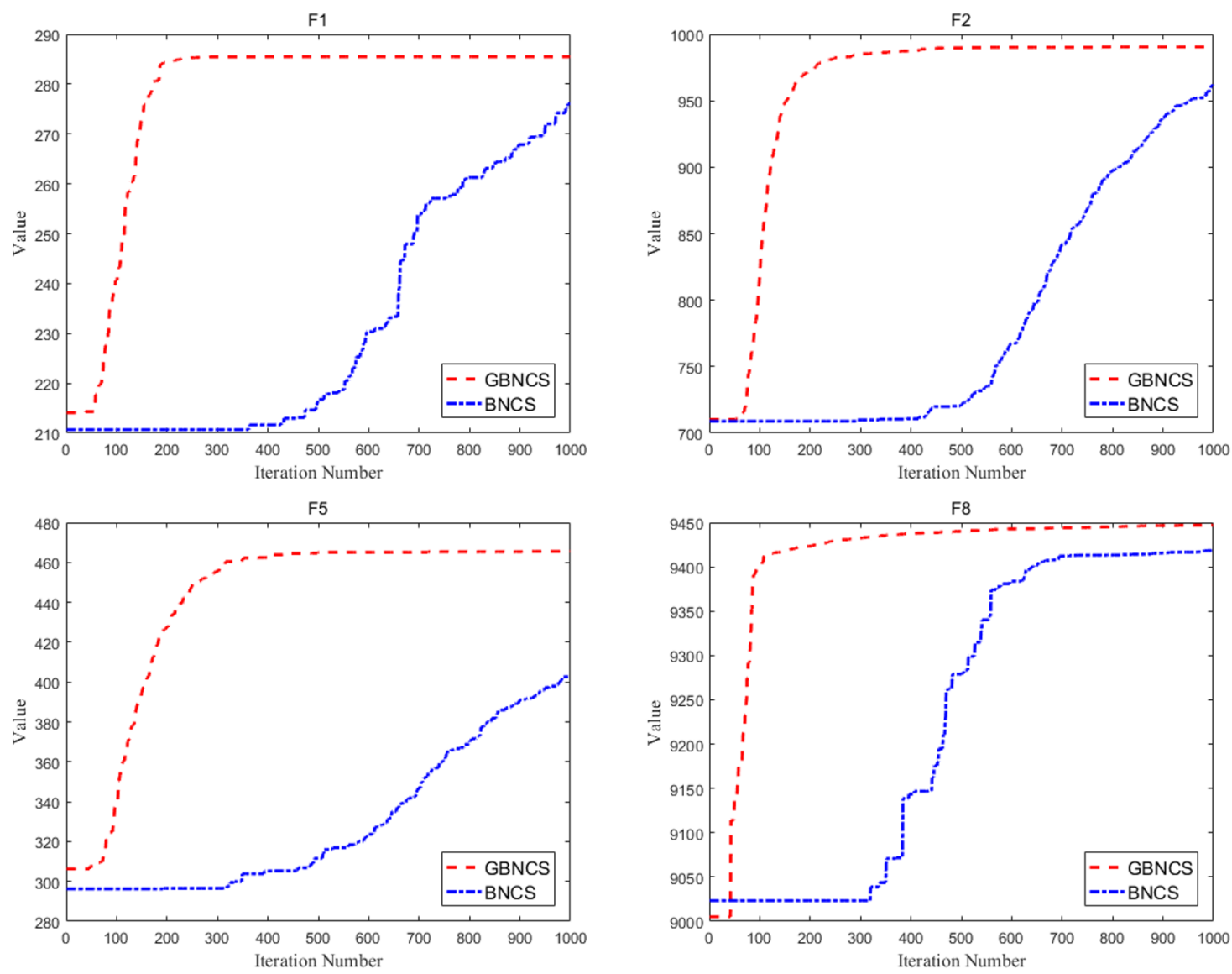

Figure 6. Convergence characteristics of BNCS and GBNCS in F1, F2, F5 and F8.

\subsection{Basic UC}

The BNCS and GBNCS methods are applied to the 10 generating unit power systems over a $24 \mathrm{~h}$ scheduling horizon. The parameters of units in 10-unit system and the load demand in $24 \mathrm{~h}$ are listed in ref. [15] and given in Tables 5 and 7.

Table 6. Numerical results of binary, negatively correlated search (BNCS) and global best inspired binary negatively search (GBNCS) approaches for Knapsack problem.

\begin{tabular}{ccccccccc}
\hline & \multirow{2}{*}{$\operatorname{dim}$} & opt.value & \multicolumn{3}{c}{ BNCS } & \multicolumn{3}{c}{ GBNCS } \\
\cline { 3 - 8 } & & & Best & Mean & Best Times & Best & Mean & Best Times \\
\hline F1 & 10 & 295 & 295 & 286.8 & 9 & 295 & 295 & 30 \\
F2 & 20 & 1024 & 1016 & 988 & 0 & 1024 & 1024 & 30 \\
F3 & 4 & 35 & 35 & 35 & 30 & 35 & 35 & 30 \\
F4 & 4 & 23 & 23 & 23 & 30 & 23 & 23 & 30 \\
F5 & 15 & 481.0694 & 481.0694 & 425.9331 & 4 & 481.0694 & 481.0694 & 30 \\
F6 & 10 & 50 & 52 & 51.6 & 21 & 50 & 50 & 30 \\
F7 & 7 & 107 & 107 & 103.6 & 27 & 107 & 107 & 30 \\
F8 & 23 & 9767 & 9752 & 9733.4 & 0 & 9767 & 9763.1 & 6 \\
F9 & 5 & 130 & 130 & 130 & 30 & 130 & 130 & 30 \\
F10 & 20 & 1025 & 1025 & 991.9 & 3 & 1025 & 1025 & 30 \\
\hline
\end{tabular}


Table 7. Load demand for $24 \mathrm{~h}$ scheduling horizon.

\begin{tabular}{cccccccc}
\hline Hour & $\boldsymbol{P}_{\text {load }}$ & Hour & $\boldsymbol{P}_{\text {load }}$ & Hour & $\boldsymbol{P}_{\text {load }}$ & Hour & $\boldsymbol{P}_{\text {load }}$ \\
\hline 1 & 700 & 7 & 700 & 13 & 1400 & 19 & 1200 \\
2 & 750 & 8 & 1150 & 14 & 1300 & 20 & 1400 \\
3 & 850 & 9 & 1200 & 15 & 1200 & 21 & 1300 \\
4 & 950 & 10 & 1300 & 16 & 1050 & 22 & 1100 \\
5 & 1000 & 11 & 1400 & 17 & 1000 & 23 & 900 \\
6 & 1100 & 12 & 1500 & 18 & 1100 & 24 & 800 \\
\hline
\end{tabular}

To comparatively study the performance of the BNCS and GBNCS, the best value, worst value, and mean value obtained by the two algorithms proposed in this paper were compared with several popular algorithms which were applied to the UC problem. These algorithms include dynamic programming [15], Lagrangian relaxation [15], the genetic algorithm (GA) [15], NS evolutionary programming (EP) [38], simulate anneal (SA) [18], improved particle swarm optimization (IPSO) [39] binary particle optimization (BPSO) [40], quantum-inspired PSO (QPSO) [41], improved quantum evolutionary algorithm (IQEA) [42], quantum-inspired evolutionary algorithm (EQA-UC) [43] binary differential evolution algorithm (BDE) [44], binary real-coded GA (brGA) [45], harmony search algorithm (HAS) [46], hybrid harmony search (HHS) [47], binary gravitational search algorithm (BGSA) [36], and binary symmetric particle swarm optimization (BSPSO) [48]. The total number of cost function evaluations (FES) was set as 30,000. Table 8 shows the experimental results over 30 independent runs.

Table 8. Comparison of BNCS and GBNCS with other approaches for 10 unit systems.

\begin{tabular}{ccccc}
\hline Method & Best & Worst & Mean & std \\
\hline DP [15] & 565825 & - & - & - \\
LR [15] & 565825 & - & - & - \\
GA [15] & 565,825 & 507,732 & 570,032 & - \\
EP [39] & 564,551 & 566,231 & 565,325 & - \\
SA [18] & 565,825 & 566,260 & 565,988 & - \\
IPSO [39] & 563,954 & 564,579 & 564,162 & - \\
BPSO [40] & 563,977 & 563,977 & 563,977 & - \\
QPSO [41] & 563,977 & 563,977 & 563,977 & - \\
IQEA [42] & 563,977 & 563,977 & 563,977 & - \\
EQA-UC [43] & 563,937 & 564,012 & 564,711 & - \\
BDE [44] & 563,937 & 564,253 & 564,088 & - \\
brGA [45] & 563,937 & - & - & - \\
HAS [46] & 563,977 & - & 564,168 & - \\
HHS [47] & 563,937 & 563,995 & 563,965 & - \\
BGSA [36] & 563,937 & 564,241 & 564,031 & - \\
BSPSO1 [48] & 563,977 & 564,018 & 563,980 & 0.002 \\
BSPSO2 [48] & 563,937 & 563,977 & 563,976 & 0.001 \\
BSPSO3 [48] & 563,937 & 563,977 & 563,973 & 0.002 \\
BSPSO4 [48] & 563,937 & 563,977 & 563,964 & 0.003 \\
BSPSO5 [48] & 563,937 & 563,977 & 563,960 & 0.003 \\
BNCS & 563,937 & 563,977 & 563,941 & 8.455 \\
GBNCS & 563,937 & 563,937 & 563,937 & 0 \\
\hline
\end{tabular}

According to Table 8, it was found that GBNCS, BNCS, BGSA, HHS, EQA-UC, BDE, and $\operatorname{BSPSO}(2-5)$ were all capable of achieving the best value 563,937 \$/day, which was also the best result among state-of-the-art UC results (some are illustrated as 563,938 \$/day.) Meanwhile, GBNCS had the best performance in mean value, and worst value. Due to the great performance in seeking optimum, GBNCS is always able to achieve the best solution in all 30 independent runs. Figure 7 shows the convergence process of GBNCS and BNCS; it was revealed that the GBNCS and BNCS 
algorithms converge within around 2000 iterations and GBNCS has better performance than BNCS in convergence.

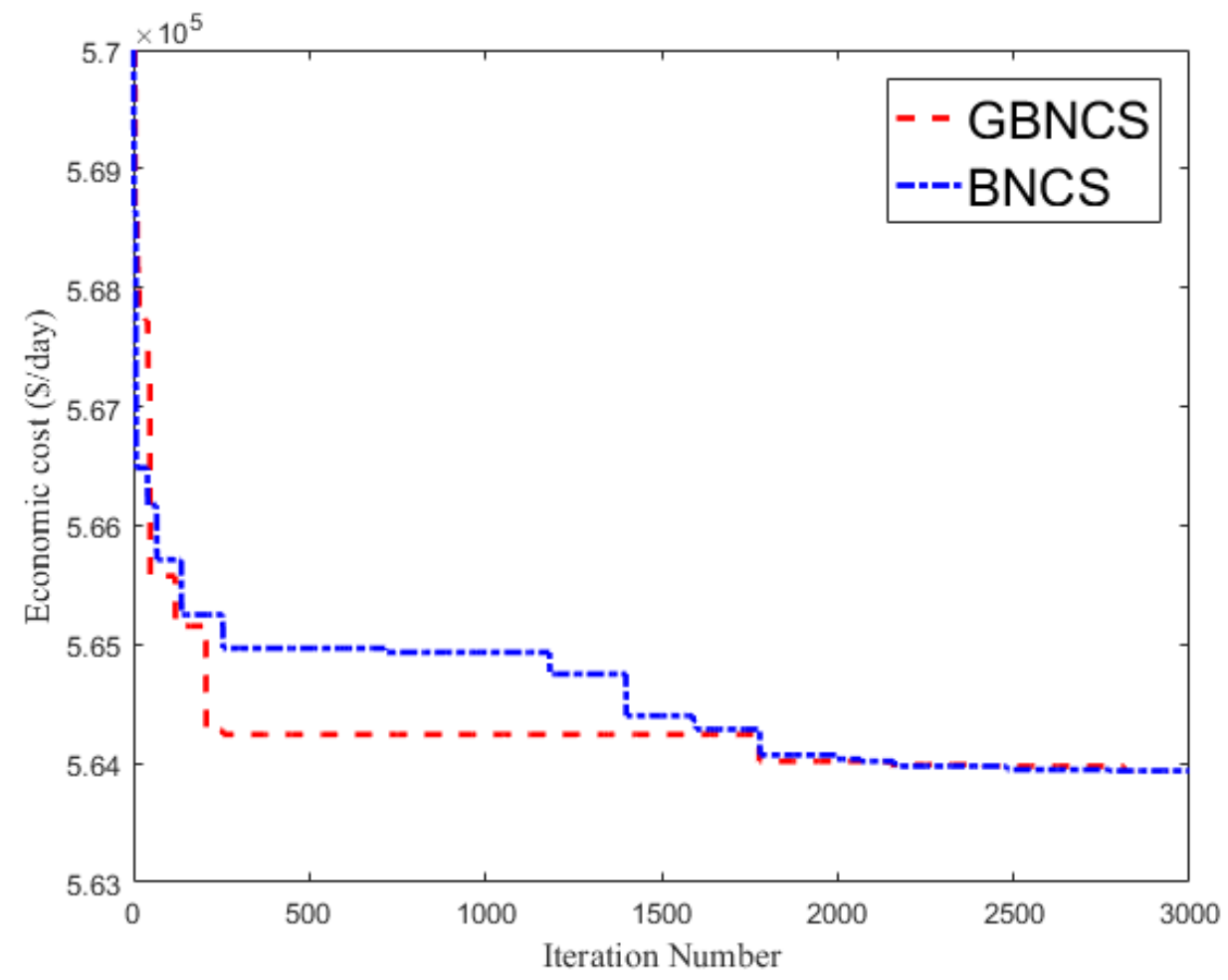

Figure 7. Convergence curve of basic unit commitment (UC) problem.

According to the above experiment, GBNCS and BNCS are competitive in dealing with the 10 unit UC benchmark problem. These two algorithms were applied to the unit commitment problem integrated with electric vehicles, which is more complex.

\subsection{UC with EVs}

Intelligent charging helps to relieve the peak load and fills the valley load, which not only reduces the fuel cost but also avoids to start-up or shut-down the generators frequently. To study the EV charging impact on the power systems, 30,000 electric vehicles were integrated into the basic 10-unit benchmark power system, and the number of electric vehicles in Equation (22) is denoted as N. The EV parameters are given in Table 9 [49], and the maximum battery capacities of these EVs were within the range of $0.01-0.25 \mathrm{MW}$. It was assumed that the average capacity was $0.015 \mathrm{MV}$ and departure state of charge $\delta$ was set to $50 \%$. The efficiency of charging is related to temperature. Here, the charging efficiency of all electric vehicles was set as $85 \%$. The total charging load and maximum charging load of electric vehicles can be obtained from the following equation, where PV is the battery capacity.

$$
P_{\text {demand }}=N * P V * \delta * \eta
$$

Table 9. Parameters of electric vehicles.

\begin{tabular}{clc}
\hline Item & Value \\
\hline Average battery capacity of an electric vehicle & $P V_{a v}(M W)$ & 0.015 \\
Maximum battery capacity of an electric vehicle & $P V_{\max }(M W)$ & 0.025 \\
Minimum battery capacity of an electric vehicle & $P V_{\min }(M W)$ & 0.010 \\
Departure state of charge $\delta$ & $50 \%$ \\
Charging efficiency $\eta$ & $85 \%$ \\
\hline
\end{tabular}


Hence, the total charging load was 191.25 MW, and the maximum charging load was $318.75 \mathrm{MW}$. Thus, the EV charging load within $24 \mathrm{~h}$ can be obtained from the total charging load and the probability distribution of four charging modes. The maximum charging load was added to the reserved constraint to consider the worst situation. Figure 8 shows the load curve after integrating electric vehicles in off-peak, peak, and EPRI modes, and basic UC loads.

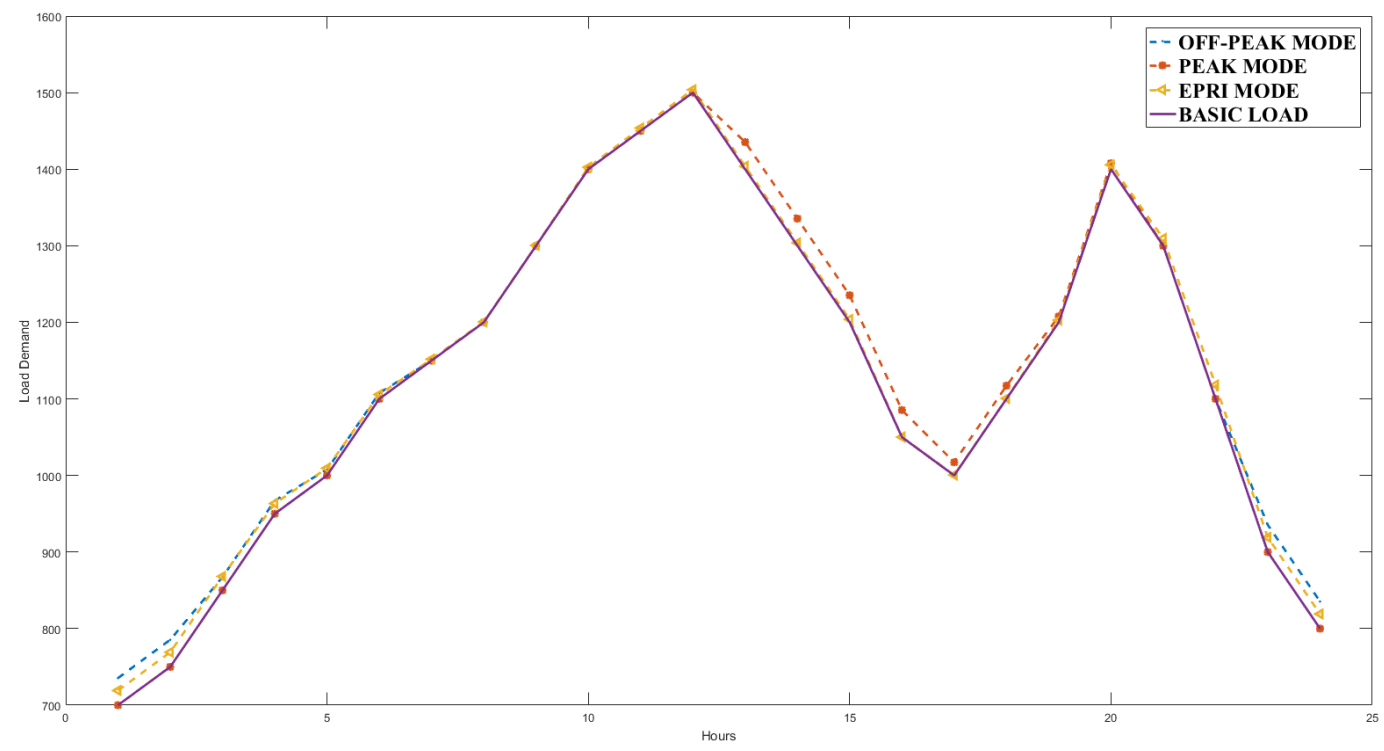

Figure 8. Load demand curve for $24 \mathrm{~h}$ scheduling horizon in different charging modes.

To investigate the impact of EV charging on power systems and test the performance of BNCS and GBNCS, these two algorithms were applied to the 10-unit UC problem integrated with EVs. The results including best values, worst values, and mean values are given in Table 10. Similar to the experiment in the basic 10 unit UC benchmark problem, the FES was set as 30,000 and the results were obtained from 30 individual runs. The population size was set to 10 .

Table 10. Performance comparison of the proposed algorithms for UC with EVs problem.

\begin{tabular}{|c|c|c|c|c|c|c|c|c|c|c|c|c|}
\hline & \multicolumn{3}{|c|}{ OFF-PEAK } & \multicolumn{3}{|c|}{ PEAK } & \multicolumn{3}{|c|}{ EPRI } & \multicolumn{3}{|c|}{ stochastic } \\
\hline & Best & Mean & Worst & Best & Mean & Worst & Best & Mean & Worst & Best & Mean & Worst \\
\hline \multirow{5}{*}{$\begin{array}{l}\text { GAH } \\
\text { [50] }\end{array}$} & \multirow{5}{*}{577,405} & \multirow{5}{*}{579,632} & \multirow{5}{*}{-} & \multirow{5}{*}{-} & \multirow{5}{*}{-} & \multirow{5}{*}{-} & \multirow{5}{*}{ - } & \multirow{5}{*}{-} & \multirow{5}{*}{ - } & 578,884 & 580,254 & - \\
\hline & & & & & & & & & & 577,932 & 580,082 & - \\
\hline & & & & & & & & & & 578,564 & 581,332 & - \\
\hline & & & & & & & & & & 580,087 & 582,258 & - \\
\hline & & & & & & & & & & 580,028 & 583,821 & - \\
\hline \multirow{5}{*}{$\begin{array}{l}\text { GAD } \\
{[50]}\end{array}$} & \multirow{5}{*}{571,085} & \multirow{5}{*}{572,577} & \multirow{5}{*}{-} & \multirow{5}{*}{-} & \multirow{5}{*}{-} & \multirow{5}{*}{-} & \multirow{5}{*}{-} & \multirow{5}{*}{-} & \multirow{5}{*}{ - } & 572,403 & 573,400 & - \\
\hline & & & & & & & & & & 572,435 & 573,184 & - \\
\hline & & & & & & & & & & 573,364 & 574,439 & - \\
\hline & & & & & & & & & & 573,543 & 574,738 & - \\
\hline & & & & & & & & & & 574,663 & 576,152 & - \\
\hline \multirow{5}{*}{ BNCS } & \multirow{5}{*}{568,372} & \multirow{5}{*}{568,373} & \multirow{5}{*}{568,374} & \multirow{5}{*}{568,894} & \multirow{5}{*}{569,120} & \multirow{5}{*}{569,210} & & & & 568,086 & 568,102 & 568,155 \\
\hline & & & & & & & & & & 568,281 & 568,283 & 568,284 \\
\hline & & & & & & & 568,201 & 568,202 & 568,210 & 568,460 & 568,764 & 569,032 \\
\hline & & & & & & & & & & 569,581 & 569,811 & 569,862 \\
\hline & & & & & & & & & & 569,648 & 569,755 & 569,834 \\
\hline & & & & & & & & & & 568,085 & 568,099 & 568,146 \\
\hline & & & & & & & & & & 568,279 & 568,282 & 568,283 \\
\hline GBNCS & 568,370 & 568,371 & 568,374 & 568,894 & 569,032 & 569,180 & 568,199 & 568,202 & 568,203 & 568,440 & 568,772 & 568,960 \\
\hline & & & & & & & & & & 569,562 & 569,806 & 569,862 \\
\hline & & & & & & & & & & 569,627 & 569,738 & 569,820 \\
\hline
\end{tabular}


As shown in Table 10, the results obtained by BNCS and GBNCS were far better than those obtained by GAD and GAH. GBNCS is always able to get the best solutions in four modes and eight scenarios. Table 10 also reflects the impact of EV charging on power systems. In the peak charging mode and the off-peak charging mode, the mean results obtained by GBNCS were 568,370 \$/day and 568,894 \$/day, respectively; peak charging costs 524 \$/day more than off-peak charging, and the reason can be found in the unit commitment schedule and reserve available for 10-unit system under peak charging mode and off-peak charging mode obtained by GBNCS given in Tables 11 and 12 . Compared with the schedule of the off-peak charging mode, the charging of EV in peak charging mode caused a sudden start-up of unit 8 at 20:00; this unit was connected to the grid for only one hour and then shut down immediately at 21:00, which greatly increased the cost. But off-peak charging mode is not the most economical way to charge: the EPRI charging mode is cheaper than the off-peak charging mode; that is because off-peak charging mode as an ideal charging mode where all the EVs are charged in the off-peak period which forms a new peak of charging, but EPRI charging mode charges most of the EVs off-peak; only a quarter of EVs are charged at home.

Table 11. Unit commitment schedule available for 10 unit system using GBNCS in Peak charging mode.

\begin{tabular}{|c|c|c|c|c|c|c|c|c|c|c|}
\hline Hour & U1 & $\mathrm{U} 2$ & U3 & U4 & U5 & U6 & U7 & U8 & U9 & U10 \\
\hline 1 & 455 & 245 & 0 & 0 & 0 & 0 & 0 & 0 & 0 & 0 \\
\hline 2 & 455 & 295 & 0 & 0 & 0 & 0 & 0 & 0 & 0 & 0 \\
\hline 3 & 455 & 370 & 0 & 0 & 25 & 0 & 0 & 0 & 0 & 0 \\
\hline 4 & 455 & 455 & 0 & 0 & 40 & 0 & 0 & 0 & 0 & 0 \\
\hline 5 & 455 & 390 & 130 & 0 & 25 & 0 & 0 & 0 & 0 & 0 \\
\hline 6 & 455 & 360 & 130 & 130 & 25 & 0 & 0 & 0 & 0 & 0 \\
\hline 7 & 455 & 410 & 130 & 130 & 25 & 0 & 0 & 0 & 0 & 0 \\
\hline 8 & 455 & 455 & 130 & 130 & 30 & 0 & 0 & 0 & 0 & 0 \\
\hline 9 & 455 & 455 & 130 & 130 & 85 & 20 & 25 & 0 & 0 & 0 \\
\hline 10 & 455 & 455 & 130 & 130 & 162 & 33 & 25 & 10 & 0 & 0 \\
\hline 11 & 455 & 455 & 130 & 130 & 162 & 73 & 25 & 10 & 0 & 10 \\
\hline 12 & 455 & 455 & 130 & 130 & 162 & 80 & 25 & 43 & 10 & 10 \\
\hline 13 & 455 & 455 & 130 & 130 & 162 & 58 & 25 & 10 & 10 & 0 \\
\hline 14 & 455 & 455 & 130 & 130 & 120 & 20 & 25 & 0 & 0 & 0 \\
\hline 15 & 455 & 455 & 130 & 130 & 45 & 20 & 0 & 0 & 0 & 0 \\
\hline 16 & 455 & 345 & 130 & 130 & 25 & 0 & 0 & 0 & 0 & 0 \\
\hline 17 & 455 & 277 & 130 & 130 & 25 & 0 & 0 & 0 & 0 & 0 \\
\hline 18 & 455 & 377 & 130 & 130 & 25 & 0 & 0 & 0 & 0 & 0 \\
\hline 19 & 455 & 443 & 130 & 130 & 25 & 0 & 25 & 0 & 0 & 0 \\
\hline 20 & 455 & 455 & 130 & 130 & 162 & 31 & 25 & 10 & 10 & 0 \\
\hline 21 & 455 & 455 & 130 & 130 & 85 & 20 & 25 & 0 & 0 & 0 \\
\hline 22 & 455 & 455 & 130 & 0 & 40 & 20 & 0 & 0 & 0 & 0 \\
\hline 23 & 455 & 450 & 0 & 0 & 25 & 0 & 0 & 0 & 0 & 0 \\
\hline 24 & 455 & 345 & 0 & 0 & 0 & 0 & 0 & 0 & 0 & 0 \\
\hline
\end{tabular}


Table 12. Unit commitment schedule available for 10 unit system using GBNCS in Off-Peak charging mode.

\begin{tabular}{ccccccccccc}
\hline Hour & U1 & U2 & U3 & U4 & U5 & U6 & U7 & U8 & U9 & U10 \\
\hline 1 & 455 & 280 & 0 & 0 & 0 & 0 & 0 & 0 & 0 & 0 \\
2 & 455 & 330 & 0 & 0 & 0 & 0 & 0 & 0 & 0 & 0 \\
3 & 455 & 387 & 0 & 0 & 25 & 0 & 0 & 0 & 0 & 0 \\
4 & 455 & 357 & 130 & 0 & 25 & 0 & 0 & 0 & 0 & 0 \\
5 & 455 & 397 & 130 & 0 & 25 & 0 & 0 & 0 & 0 & 0 \\
6 & 455 & 367 & 130 & 130 & 25 & 0 & 0 & 0 & 0 & 0 \\
7 & 455 & 410 & 130 & 130 & 25 & 0 & 0 & 0 & 0 & 0 \\
8 & 455 & 455 & 130 & 130 & 30 & 0 & 0 & 0 & 0 & 0 \\
9 & 455 & 455 & 130 & 130 & 85 & 20 & 25 & 0 & 0 & 0 \\
10 & 455 & 455 & 130 & 130 & 162 & 33 & 25 & 10 & 0 & 0 \\
11 & 455 & 455 & 130 & 130 & 162 & 73 & 25 & 10 & 10 & 0 \\
12 & 455 & 455 & 130 & 130 & 162 & 80 & 25 & 43 & 10 & 10 \\
13 & 455 & 455 & 130 & 130 & 162 & 33 & 25 & 10 & 0 & 0 \\
14 & 455 & 455 & 130 & 130 & 85 & 20 & 25 & 0 & 0 & 0 \\
15 & 455 & 455 & 130 & 130 & 30 & 0 & 0 & 0 & 0 & 0 \\
16 & 455 & 310 & 130 & 130 & 25 & 0 & 0 & 0 & 0 & 0 \\
17 & 455 & 260 & 130 & 130 & 25 & 0 & 0 & 0 & 0 & 0 \\
18 & 455 & 340 & 130 & 130 & 25 & 20 & 0 & 0 & 0 & 0 \\
19 & 455 & 440 & 130 & 130 & 25 & 20 & 0 & 0 & 0 & 0 \\
20 & 455 & 455 & 130 & 130 & 162 & 33 & 25 & 10 & 0 & 0 \\
21 & 455 & 455 & 130 & 130 & 85 & 20 & 25 & 0 & 0 & 0 \\
22 & 455 & 455 & 130 & 0 & 35 & 0 & 25 & 0 & 0 & 0 \\
23 & 455 & 455 & 0 & 0 & 25 & 0 & 0 & 0 & 0 & 0 \\
24 & 455 & 355 & 0 & 0 & 25 & 0 & 0 & 0 & 0 & 0 \\
\hline
\end{tabular}

Figure 9 shows the computation time for solving the unit commitment integrated with electric vehicle problem and knapsack problem. GBNCS only uses about half of the calculation time of BNCS in all these four modes. Furthermore, when solving the knapsack problem, the calculation time of GBNCS is almost one tenth that of BNCS. The efficiency of GBNCS is more obvious when dealing with simple optimization problems, such as the knapsack problem. That is because the object function of the knapsack problem is much simpler then UC's, so when dealing with knapsack problem, the objective function evaluation time is much less than the Bhattacharyya distance calculation time. GBNCS optimized the problem with significantly reduced computation time; therefore, the efficiency of the algorithm was greatly enhanced.
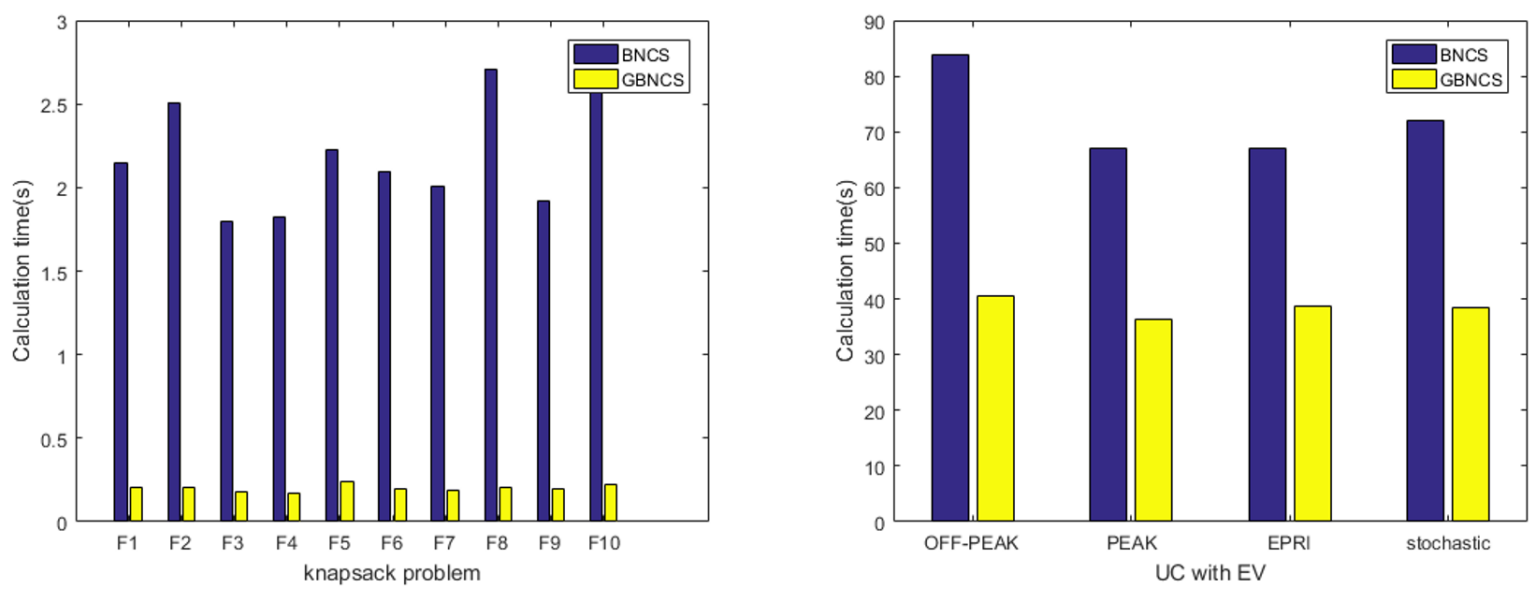

Figure 9. Computational time of BNCS and GBNCS for the knapsack problem and UC with EVs problem. 


\section{Conclusions}

This paper has proposed a unit commitment problem integrated with electric vehicles. Two algorithms, namely, the binary negatively correlated search and the global best inspired, binary, negatively correlated search have been proposed, for which a rounding transfer function to convert real-valued variables to binary ones was proposed. Furthermore, the GBNCS uses the global best information to improve the efficiency and convergence of the algorithm. The proposed algorithm was first tested on a benchmark knapsack problem and a basic unit commitment problem, and then applied to the unit commitment integrated with an electric vehicle model.

To comprehensively study the charging model, four charging modes were used to simulate different charging scenarios. The results confirmed that the proposed BNCS and GBNCS are very competitive in dealing with the traditional knapsack benchmark problem and the UCEV problem. The GBNCS is not only able to achieve good quality solutions to dispatch the committed units economically but also has great performance in convergence and computation time.

Our future work will consider other practical constraints of EV charging and discharging in real-world applications.

Author Contributions: Q.N. proposed the idea; K.J. and Z.Y. collected the data, designed the experiments and implemented the experiments; Q.N., K.J. analyzed the data and wrote the paper.

Funding: This paper was supported by the National Natural Science Foundation of China (61773252) and Natural Science Foundation of Guangdong Province (2018A030310671).

Conflicts of Interest: The authors declare no conflict of interest.

\section{References}

1. Moss, R.H.; Edmonds, J.A.; Hibbard, K.A. The next generation of scenarios for climate change research and assessment. Nature 2010, 463, 747-756. [CrossRef] [PubMed]

2. U.S. Energy Information Administration (EIA). International Energy Outlook 2019. Available online: https: //www.eia.gov/ (accessed on 1 July 2019).

3. Mohammadi, F. Hybridization of an Electric Vehicle Using Lithium-ion Batteries. In Proceedings of the 1st International Conference on Modern Approaches in Engineering Science(ICMAES), Tbilisi, Georgia, 28-29 August 2018.

4. Mohammadi, F. Electric Vehicle Battery Market Analysis: Lead Acid. In Proceedings of the 9th Iranian Conference on Electrical and Electronics Engineering (ICEEE), Gonabad, Iran, 28-29 August 2018.

5. Mohammadi, F. Electric Vehicle Battery Market Analysis: Nickel Metal Hydride. In Proceedings of the 9th Iranian Conference on Electrical and Electronics Engineering (ICEEE), Gonabad, Iran, 28-29 August 2018.

6. Mohammadi, F. Electric Vehicle Battery Market Analysis: Lithium-ion. In Proceedings of the 1st International Conference on Modern Approaches in Engineering Science (ICMAES), Tbilisi, Georgia, 28-29 August 2018.

7. Fernández, R.A. A more realistic approach to electric vehicle contribution to greenhouse gas emissions in the city. J. Clean. Prod. 2018, 172, 949-959. [CrossRef]

8. Mohammadi, F. Design and Electrification of an Electric Vehicle Using Lithium-ion Batteries. In Proceedings of the 1rd International Conference on Electrical Engineering, Zurich, Switzerland, 28-29 August 2018.

9. Clement-Nyns, K.; Haesen, E.; Driesen, J. The Impact of Charging Plug-In Hybrid Electric Vehicles on a Residential Distribution Grid. IEEE Trans. Power Systems 2010, 25, 371-380. [CrossRef]

10. Fernandes, C.; Frías, P.; Latorre, J.M. Impact of vehicle-to-grid on power system operation costs: The Spanish case study. Appl. Energy 2012, 96, 194-202. [CrossRef]

11. Zhou, N.; Xiong, X.; Xiong, Q. Probability Model and Simulation Method of Electric Vehicle Charging Load on Distribution Network. J. Power Sources 2014, 42, 879-888. [CrossRef]

12. Go, H.; Cho, I.; Kim, C. A Study on EV Charging Scheme Using Load Control. J. Electr. Eng. Technol. 2017, 12, 1789-1797.

13. Snyder, W.; Powell, D.; Rayburn, J. Dynamic programming approach to unit commitment. IEEE Trans. Power Syst. 1987, 2, 339-348. [CrossRef] 
14. Jiang, Q.; Zhou, B.; Zhang, M. Parallel augment Lagrangian Relaxation method for transient stability constrained unit commitment. IEEE Trans. Power Syst. 2013, 28, 1140-1148. [CrossRef]

15. Kazarlis, S.A.; Bakirtzis, A.G.; Petridis, V. A genetic algorithm solution to the unit commitment problem. IEEE Trans. Power Syst. 1996, 11, 83-92. [CrossRef]

16. Sasaki, H.; Watanabe, M.; Kubokawa, J. A solution method of unit commitment by artificial neural networks. IEEE Trans. Power Syst. 1992, 7, 974-981. [CrossRef]

17. Rajan, C.C.A.; Mohan, M.R. An evolutionary programming-based tabu search method for solving the unit commitment problem. IEEE Trans. Power Syst. 2004, 19, 577-584. [CrossRef]

18. Simopoulos, D.N.; Kavatza, S.D.; Vournas, C.D. Unit Commitment by an Enhanced Simulated Annealing Algorithm. 2006 IEEE PES Power Syst. Conf. Expo. 2006, 1, 193-201.

19. Jiang, R.; Wang, J.; Guan, Y. Robust unit commitment with wind power and pumped storage hydro. IEEE Trans. Power Syst. 2012, 27, 800-810. [CrossRef]

20. Alemany, J.; Magnago, F.; Moitre, D. Symmetry issues in mixed integer programming based Unit Commitment. Int. J. Electr. Power Energy Syst. 2014, 54, 86-90. [CrossRef]

21. Logenthiran, T.; Srinivasan, D. Particle Swarm Optimization for unit commitment problem. 2010 IEEE 11th Int. Conf. Probabilistic Methods Appl. Power Syst. 2010, 1, 642-647.

22. Saber, A.Y.; Venayagamoorthy, G.K. Intelligent unit commitment with vehicle-to-grid-A cost-emission optimization. Electr. Power Compon. Syst. 2010, 195, 898-911. [CrossRef]

23. Foley, A.; Tyther, B.; Calnan, P.; Gallachoir, B.O. Impacts of electric vehicle charging under electricity market operations. Appl. Energy 2013, 101, 93-102. [CrossRef]

24. Ma, H.; Yang, Z.; You, P. Multi-objective biogeography-based optimization for dynamic economic emission load dispatch considering plug-in electric vehicles charging. J. Sol. Energy Res. 2018, 3, 293-299. [CrossRef]

25. Liu, C.; Wang, J.; Botterud, A. Assessment of Impacts of PHEV Charging Patterns on Wind-Thermal Scheduling by Stochastic Unit Commitment. IEEE Trans. Smart Grid 2012, 3, 675-683. [CrossRef]

26. Mohammadi, F. Design, Analysis, and Electrification of a Solar-Powered Electric Vehicle. J. Solar Energy Res. 2018, 3.

27. Yang, Z.; Li, K.; Niu, Q. A comprehensive study of economic unit commitment of power systems integrating various renewable generations and plug-in electric vehicles. Energy Convers. Manag. 2017, 132, 460-481. [CrossRef]

28. Mohammadi, F.; Nazri, G.-A.; Nazri, M. A Bidirectional Power Charging Control Strategy for Plug-in Hybrid Electric Vehicles. Sustainability 2019, 11, 4317. [CrossRef]

29. Yang, L.; Li, K.; Xu, X. A hybrid meta-heuristic method for unit commitment considering flexible charging and discharging of plug-in electric vehicles. In Proceedings of the 2016 IEEE Congress on Evolutionary Computation (CEC), Vancouver, BC, Canada, 24-29 July 2016; pp. 2014-2020.

30. Tang, K.; Yang, P.; Yao, X. Negatively Correlated Search. IEEE J. Select. Areas Commun. 2016, 34, 542-550. [CrossRef]

31. EPRI executive summary: Environmental assessment of plug-in hybrid electric vehicles. In United States air quality Analysis Based on AEO-2006 Assumptions for 2030; Electric Power Research Institute: Palo Alto, CA, USA, 2007; Volume 2.

32. Kailath, $\mathrm{T}$. The divergence and Bhattacharyya distance measures in signal selection. IEEE Trans. Commun. Technol. 1967, 15, 52-60. [CrossRef]

33. Niu, Q.; Jiang, K.; Liu, B. A Novel Binary Negatively Correlated Search for Wind Farm Layout Optimization. In Proceedings of the 2019 IEEE Congress on Evolutionary Computation (CEC), Wellington, New Zealand, 10-13 June 2019; pp. 191-196.

34. Brier, E.; Clavier, C.; Olivier, F. Correlation power analysis with a leakage model. Lect. Notes Comput. Sci. 2004, 3156, 16-29.

35. Rechenberg, I. Evolutionsstrategie Optimierung Technischer Systeme nach Prinzipien der Biologischen Evolution. Ph.D. Thesis, Universität zu Berlin, Berlin, Germany, 1973.

36. Yuan, X.; Ji, B.; Zhang, S. A new approach for unit commitment problem via binary gravitational search algorithm. Appl. Soft Comput. 2014, 22, 249-260. [CrossRef]

37. Zou, D.; Gao, L.; Li, S.; Wu, J. Solving 0-1 knapsack problem by a novel global harmony search algorithm. Appl. Soft Comput. 2011, 11, 1556-1564. [CrossRef] 
38. Juste, K.A.; Kita, H.; Tanaka, E.; Hasegawa, J. An evolutionary programming solution to the unit commitment problem. IEEE Trans. Power Syst. 1999, 14, 1452-1459. [CrossRef]

39. Zhao, B.; Guo, C.; Bai, B.; Cao, Y. An improved particle swarm optimization algorithm for unit commitment. Int. J. Electr. Power Energy Syst. 2006, 28, 482-490. [CrossRef]

40. Gaing, Z. Discrete particle swarm optimization algorithm for unit commitment. 2003 IEEE Power Eng. Soc. Gener. Meet. 2003, 1, 418-424.

41. Jeong, Y.; Park, J.; Jang, S.; Lee, K.Y. A New Quantum-Inspired Binary PSO: Application to Unit Commitment Problems for Power Systems. IEEE Trans. Power Syst. 2010, 25, 1486-1495. [CrossRef]

42. Jeong, Y.; Park, J.; Shin, J.; Lee, K.Y. A thermal unit commitmentapproach using an improved quantum evolutionary algorithm. Electr. Power Compon. Syst. 2009, 37, 770-786. [CrossRef]

43. Lau, T.W.; Chung, C.Y.; Wong, K.P.; Chung, T.S.; Ho, S.L. Quantum-Inspired Evolutionary Algorithm Approach for Unit Commitment. IEEE Trans. Power Syst. 2009, 24, 1503-1512. [CrossRef]

44. Ghasemi, A.; Farsangi, M.M.; Nezamabadi-pour, H. Unit commitment scheduling using binary differential evolution algorithm. OPSEARCH 2009, 46, 108-122. [CrossRef]

45. Datta, D. Unit commitment problem with ramp rate constraint using a binary-real-coded genetic algorithm. Appl. Soft Comput. 2013, 13, 3873-3883. [CrossRef]

46. Afkousi-Paqaleh, M.; Rashidinejad, M.; Pourakbari-Kasmaei, M. An implementation of harmony search algorithm to unit commitment problem. Appl. Soft Comput. 2010, 92, 212-225. [CrossRef]

47. Kamboj, V.K.; Bath, S.K.; Dhillon, J.S. Hybrid HS-random search algorithm considering ensemble and pitch violation for unit commitment problem. Neural Comput. Appl. 2017, 28, 1123-1148. [CrossRef]

48. Yang, Z.; Li, K.; Feng, S.; Niu, Q. A binary symmetric based hybrid meta-heuristic method for solving mixed integer unit commitment problem integrating with significant plug-in electric vehicles. Energy 2019, 170, 889-905. [CrossRef]

49. Ehsan, T.; Masoud, R.; Amir, A. Evaluation of plug-in electric vehicles impact on cost-based unit commitment. Power Sources 2014, 248, 545-552.

50. Zhang, L.; Niu, Q.; Yang, Z. Integration of electric vehicles charging in unit commitment. Int. J. Comput. Sci. Electron. Eng. 2015, 3, 22-27.

(C) 2019 by the authors. Licensee MDPI, Basel, Switzerland. This article is an open access article distributed under the terms and conditions of the Creative Commons Attribution (CC BY) license (http://creativecommons.org/licenses/by/4.0/). 